Bull. Austral. Math. Soc.

Vol. 46 (1992) [525-528]

\title{
AN INTERNAL CHARACTERISATION OF STRONGLY REGULAR RINGS
}

\section{LuOSheng Huang and Weimin Xue}

We show that a right duo ring $R$ is strongly regular if and only if for each ideal $I$ of $R$, the coset product of $I$ in the factor ring $R / I$ is the same as their set product.

In this note a ring means an associative ring with identity. If $I$ is an ideal of a ring $R$, then the coset product of $I$ in the factor ring $R / I$ is defined as

$$
\left(r_{1}+I\right)\left(r_{2}+I\right)=r_{1} r_{2}+I
$$

where $r_{1}, r_{2} \in R$. Since $r_{1}+I$ and $r_{2}+I$ are subsets of $R$, we can define their set product as

$$
\left(r_{1}+I\right) \circ\left(r_{2}+I\right)=\left\{\left(r_{1}+i_{1}\right)\left(r_{2}+i_{2}\right) \mid i_{1}, i_{2} \in I\right\} \text {. }
$$

We always have that $\left(r_{1}+I\right) \circ\left(r_{2}+I\right) \subseteq\left(r_{1}+I\right)\left(r_{2}+I\right)$, since $I$ is an ideal. We say that the ideal $I$ is a good ideal in case

$$
\left(r_{1}+I\right) \circ\left(r_{2}+I\right)=\left(r_{1}+I\right)\left(r_{2}+I\right)
$$

for any $r_{1}, r_{2} \in R$. A necessary condition for an ideal $I$ to be good is that $I=I \circ I$, so the ideal $2 \mathbb{Z}_{4}$ of $\mathbb{Z}_{4}=\mathbb{Z} / 4 \mathbb{Z}$ is not a good ideal. We also say that a ring $R$ is a good ring in case each ideal is good. Since the two trivial ideals are always good, each simple ring is a good ring. For this reason we consider right duo rings (after E.H. Feller), that is, rings whose right ideals are ideals. Our main result states that a right duo ring is good if and only if it is strongly regular.

LEMMA 1. Let $R$ be a right duo ring. If $I$ is a finitely generated right ideal then the following are equivalent:

(1) $I=e R$ for some idempotent $e \in R$;

(2) $I$ is a good ideal;

(3) $I=I \circ I$;

(4) $I=I^{2}$, where $I^{2}$ is defined as usual, that is, $I^{2}=\left\{\sum_{k} i_{k} j_{k} \mid i_{k}, j_{k} \in I\right\}$.

Received 4 December 1991

Copyright Clearance Centre, Inc. Serial-fee code: 0004-9729/92 \$A2.00+0.00. 
Proof: (1) $\Rightarrow$ (2). By [2, Theorem 1.3], each idempotent of $R$ belongs to the centre of $R$. Let $L=(1-e) R$, then $R=I \oplus L$ where $I$ and $L$ are ideals. Take $r_{1}, r_{2} \in R$ and $i \in I$, then $r_{1}=j_{1}+l_{1}$ and $r_{2}=j_{2}+l_{2}$ for some $j_{1}, j_{2} \in I$ and $l_{1}, l_{2} \in L$. Since $I=e R$ is generated by a central idempotent $e$, we have

$$
I=I \circ I=\left(j_{1}+I\right) \circ\left(j_{2}+I\right) \text {. }
$$

Now $j_{1} j_{2}+i \in I$, so there are $j_{1}^{\prime}, j_{2}^{\prime} \in I$ such that $j_{1} j_{2}+i=\left(j_{1}+j_{1}^{\prime}\right)\left(j_{2}+j_{2}^{\prime}\right)$. Then $i=j_{1} j_{2}^{\prime}+j_{1}^{\prime} j_{2}+j_{1}^{\prime} j_{2}^{\prime}=r_{1} j_{2}^{\prime}+j_{1}^{\prime} r_{2}+j_{1}^{\prime} j_{2}^{\prime}$ and hence $r_{1} r_{2}+i=\left(r_{1}+j_{1}^{\prime}\right)\left(r_{2}+j_{2}^{\prime}\right) \epsilon$ $\left(r_{1}+I\right) \circ\left(r_{2}+I\right)$. Therefore $\left(r_{1}+I\right)\left(r_{2}+I\right)=r_{1} r_{2}+I \subseteq\left(r_{1}+I\right) \circ\left(r_{2}+I\right)$, and then $I$ is a good ideal.

(2) $\Rightarrow$ (3). Trivial.

(3) $\Rightarrow$ (4). Since $I=I \circ I \subseteq I^{2} \subseteq I$, we have $I^{2}=I$.

(4) $\Rightarrow$ (1). By $[1$, Proposition 4].

One notes that in the above lemma the implications (2) $\Rightarrow(3) \Rightarrow(4)$ hold for an arbitrary ideal $I$ of an arbitrary ring $R$, but the right duo assumption is essential for the implications (4) $\Rightarrow(3),(4) \Rightarrow(1)$ and $(2) \Rightarrow(1)$, as shown by the following two examples.

EXAMPLE 2: The noetherian ring $R=\left[\begin{array}{cc}\mathbb{Z} & 2 \mathbb{Z} \\ \mathbb{Z} & \mathbb{Z}\end{array}\right]$ has an ideal $I=\left[\begin{array}{cc}2 \mathbb{Z} & 2 \mathbb{Z} \\ \mathbb{Z} & \mathbb{Z}\end{array}\right]$. One sees that $I=I^{2}$. But we note that $I \neq I \circ I$, since $\left[\begin{array}{ll}2 & 4 \\ 0 & 1\end{array}\right] \in I$ but $\left[\begin{array}{ll}2 & 4 \\ 0 & 1\end{array}\right] \notin I \circ I$. It follows that $I$ is not a good ideal. One also checks that $I \neq e R$ and $I \neq R e$ for any idempotent $e \in I$.

Example 3. Let $F$ be a field and $R=\left[\begin{array}{ll}F & F \\ 0 & F\end{array}\right]$. It is easy to see that $I=\left[\begin{array}{ll}0 & F \\ 0 & F\end{array}\right]$ is an ideal of $R$ and $I=I \circ I$. To show that $I$ is a good ideal, we may assume that $r_{1}=\left[\begin{array}{ll}a & 0 \\ 0 & 0\end{array}\right], r_{2}=\left[\begin{array}{ll}b & 0 \\ 0 & 0\end{array}\right] \in R$, and $i=\left[\begin{array}{ll}0 & c \\ 0 & d\end{array}\right] \in I$. We take $j=\left[\begin{array}{ll}0 & 0 \\ 0 & 1\end{array}\right] \in I$ and have that $r_{1} r_{2}+i=\left(r_{1}+i\right)\left(r_{2}+j\right) \in\left(r_{1}+I\right) \circ\left(r_{2}+I\right)$. This proves that $I$ is a good ideal. But $I \neq e R$ for any idempotent $e \in I$.

Similarly, the ideal $L=\left[\begin{array}{ll}F & F \\ 0 & 0\end{array}\right]$ is also a good ideal. Now $I \cap L=I L=\left[\begin{array}{ll}0 & F \\ 0 & 0\end{array}\right]$, which is not a good ideal since $\left[\begin{array}{ll}0 & F \\ 0 & 0\end{array}\right] \neq\left[\begin{array}{ll}0 & F \\ 0 & 0\end{array}\right] \circ\left[\begin{array}{ll}0 & F \\ 0 & 0\end{array}\right]$. We conclude that the intersection or the product of two good ideals need not be a good ideal.

The next result asserts that to show a right duo ring is good we only need to check the principal right ideals.

LEмма 4. The following are equivalent for a right duo ring $R$ :

(1) $R$ is a good ring; 
(2) each finitely generated right ideal is a good ideal;

(3) each principal right ideal is a good ideal.

PRoOF: It remains to verify (3) $\Rightarrow$ (1). So let $I$ be an ideal of $R, r_{1}, r_{2} \in R$, and $i \in I$. Since the ideal $i R$ is a principal right ideal, we have $r_{1} r_{2}+i \in\left(r_{1}+i R\right) \circ$ $\left(r_{2}+i R\right) \subseteq\left(r_{1}+I\right) \circ\left(r_{2}+I\right)$. Hence $r_{1} r_{2}+I \subseteq\left(r_{1}+I\right) \circ\left(r_{2}+I\right)$, and then $I$ is a good ideal.

Recall that a ring $R$ is von Neuman regular if each principal right (or left) ideal of $R$ is generated by an idempotent, and $R$ is strongly regular if and only if it is regular and right duo (since idempotents in a right duo ring are central by [2, Theorem 1.3]). The following main result follows from Lemmas 1 and 4 , which gives an internal characterisation of strongly regular rings.

Theorem 5. Let $R$ be a right duo ring. Then $R$ is (strongly) regular if and only if it is a good ring.

Since any simple ring is a good ring, a good ring need not be regular. We do not know whether or not a regular ring must be a good ring.

Recall that a regular right (or left) noetherian ring is semisimple, and a semisimple right duo ring is a finite direct sum of division rings. Thus we have

Corollary 6. Let $R$ be a right duo ring. If $R$ is either right or left noetherian, then $R$ is a good ring if and only if it is a finite direct sum of division rings.

In particular, we have

COROLlaRY 7. The ring $\mathbb{Z}_{n}$ is a good ring if and only if $n$ has a square free factorisation.

It is known that a right duo ring $R$ is regular if and only if each simple right (left) $R$-module is injective (see [4, Theorem 1.3], or [3, Theorem 4.10]).

CoRollary 8. A right duo ring $R$ is a good ring if and only if each simple right (left) $R$-module is injective.

As we mention in the introduction, a necessary condition for an ideal $I$ to be good is that $I=I \circ I$, but we do not know whether or not this condition is also sufficient. Our Lemma 1 and the following concluding proposition give some positive answers.

Proposition 9. Let $J$ be the (Jacobson) radical of a local ring $R$. If $J=$ $J$ o $J$, then $J$ is a good ideal.

Proof: Let $r_{1}, r_{2} \in R$ and $j \in J$. Since $J=J \circ J$ we may assume that $r_{2} \notin J$. Then $r_{2}$ is invertible, since $R$ is local. So we have $r_{1} r_{2}+j=\left(r_{1}+j r_{2}^{-1}\right)\left(r_{2}+0\right) \in$ $\left(r_{1}+J\right) \circ\left(r_{2}+J\right)$. Therefore $r_{1} r_{2}+J \subseteq\left(r_{1}+J\right) \circ\left(r_{2}+J\right)$, and $J$ is a good ideal. $\square$ 


\section{REFERENCES}

[1] A.W. Chatters and Weimin Xue, 'On right duo p.p. rings', Glasgow Math. J. 32 (1990), 221-225.

[2] R.C. Courter, 'Finite dimensional right duo algebras are duo', Proc. Amer. Math. Soc. 84 (1982), 157-162.

[3] M.B. Rege, 'On von Neumann regular rings and $S F$-rings', Math. Japon. 31 (1986), 927-936.

[4] R. Yue Chi Ming, 'On von Neumann regular rings $V$ ', Math. J. Okayama Univ. 22 (1980), 151-160.

Department of Mathematics

Fujian Normal University

Fuzhou

Fujian 350007

Peoples Republic of China
Current address:

Department of Mathematics

University of Iowa

Iowa City IA 52242

United States of America 